\title{
Compressive Sensing Approach in the Hermite Transform Domain
}

\author{
Srdjan Stanković, Ljubiša Stanković, and Irena Orović \\ University of Montenegro, Faculty of Electrical Engineering, Dzordza Vasingtona bb, 81000 Podgorica, Montenegro
}

Correspondence should be addressed to Irena Orović; irenao@ac.me

Received 20 August 2015; Accepted 16 November 2015

Academic Editor: Yuqiang Wu

Copyright (C) 2015 Srdjan Stanković et al. This is an open access article distributed under the Creative Commons Attribution License, which permits unrestricted use, distribution, and reproduction in any medium, provided the original work is properly cited.

\begin{abstract}
Compressive sensing has attracted significant interest of researchers providing an alternative way to sample and reconstruct the signals. This approach allows us to recover the entire signal from just a small set of random samples, whenever the signal is sparse in certain transform domain. Therefore, exploring the possibilities of using different transform basis is an important task, needed to extend the field of compressive sensing applications. In this paper, a compressive sensing approach based on the Hermite transform is proposed. The Hermite transform by itself provides compressed signal representation based on a smaller number of Hermite coefficients compared to the signal length. Here, it is shown that, for a wide class of signals characterized by sparsity in the Hermite domain, accurate signal reconstruction can be achieved even if incomplete set of measurements is used. Advantages of the proposed method are demonstrated on numerical examples. The presented concept is generalized for the short-time Hermite transform and combined transform.
\end{abstract}

\section{Introduction}

The Hermite polynomials and Hermite functions have attracted the attention of researchers in various fields of engineering and signal processing [1-7], such as in quantum mechanics (harmonic oscillators), ultra-high band telecommunication channels, and ECG data compression using Hermite functions representation of the QRS complexes. A set of Hermite functions forming an orthonormal basis is suitable for approximation, classification, and data compression tasks [3]. Since the Hermite functions are eigenfunctions of the Fourier transform, time and frequency spectra are simultaneously approximated. Here, we are especially interested in a class of signals that are sparse in the Hermite transform domain. Note that, generally, such signals are not sparse in the Fourier transform domain. In the light of compressive sensing (CS) theory [8-12], we propose the method for efficient reconstruction of signals from its incomplete set of samples using the Hermite transform. The number of Hermite functions used in this approach is much smaller compared to the original length of the signal. The proposed approach is useful in the applications where the significant information is missing and the total signal reconstruction is required.
Note that the large amount of missing signal samples may occur as a consequence of the compressed sampling strategy but also as a consequence of discarding damaged signal parts [13-15]. The theory is illustrated through examples showing that the Hermite transform based CS for certain types of signals can outperform the Fourier transform related reconstructions. Furthermore, in analogy with the timefrequency analysis based on the Fourier transform, the shorttime Hermite transform is defined as a linear representation that reveals the local behavior of windowed signal parts. If the signal components are of short duration, then the short-time Hermite transform is more suitable for compressive sensing than the standard Hermite transform. Finally, the possibility of combining different transforms depending on the signal characteristics is explored.

The paper is organized as follows. The theory behind the Hermite transform and the fast method for Hermite coefficients calculation is given in Section 2. The formulation of compressive sensing approach in the Hermite transform domain is given in Section 3. The possibilities to exploit other sparsity domains based on the short-time Hermite transform and combined transform are presented in Section 4. The experimental evaluation of the proposed approach is given 
in Section 4, while the concluding remarks are given in Section 5.

\section{Hermite Transform}

The Hermite functions provide good localization and the compact support in both time and frequency domain [4]. The $i$ th order Hermite function is defined as follows:

$$
\psi_{i}(t)=\frac{(-1)^{i} e^{t^{2} / 2}}{\sqrt{2^{i} i ! \sqrt{\pi}}} \cdot \frac{d^{i}\left(e^{-t^{2}}\right)}{d t^{i}} .
$$

The Hermite functions provide an orthonormal basis set for an optimal representation of different signals using the fewest number of basis functions. Signal expansion into Hermite functions, known as the Hermite transform, has been used for both $1 \mathrm{D}$ and $2 \mathrm{D}$ signals in various applications. The Hermite expansion for a signal $f(t)$ can be defined as follows:

$$
\widehat{f}(t)=\sum_{i=0}^{N-1} C_{i} \psi_{i}(t)
$$

where $\psi_{i}(t)$ are the Hermite functions and $N$ is the number of functions used for the approximation. The number of Hermite functions $N$ could be usually much smaller than the number of signal samples $M(N \leq M)$. The Hermite coefficients can be defined by using the Hermite polynomials as follows:

$$
C_{i}=\frac{1}{\sqrt{2^{i} i ! \sqrt{\pi}}} \int_{-\infty}^{\infty} e^{-t^{2}}\left(f(t) e^{t^{2}}\right) H_{i}(t) d t,
$$

where

$$
H_{i}(t)=(-1)^{i} e^{t^{2}} \frac{d^{i}\left(e^{-t^{2}}\right)}{d t^{i}}
$$

represents the Hermite polynomial. An efficient procedure for calculation of Hermite coefficients can be done by applying the Gauss-Hermite quadrature [5]:

$$
\begin{aligned}
& C_{i} \\
& =\frac{1}{\sqrt{2^{i} i ! \sqrt{\pi}}} \sum_{m=1}^{M} \frac{2^{M-1} M ! \sqrt{\pi}}{M^{2} H_{M-1}^{2}\left(t_{m}\right)}\left(f\left(t_{m}\right) e^{t_{m}^{2} / 2}\right) H_{i}\left(t_{m}\right),
\end{aligned}
$$

where $t_{m}$ are zeros of Hermite polynomials. By using the Hermite functions instead of polynomials, a simplified expression is obtained:

$$
C_{i} \approx \frac{1}{M} \sum_{m=1}^{M} \mu_{M-1}^{i}\left(t_{m}\right) f\left(t_{m}\right) .
$$

The constants $\mu_{M-1}^{i}\left(t_{m}\right)$ are calculated as follows:

$$
\mu_{M-1}^{i}\left(t_{m}\right)=\frac{\psi_{i}\left(t_{m}\right)}{\left(\psi_{M-1}\left(t_{m}\right)\right)^{2}} .
$$

\section{Compressive Sensing Formulation in the Hermite Transform Domain}

Generally, the compressive sensing scenarios are focused on the new sampling strategy, which results in a large number of randomly missing samples compared to the standard sampling methods [8]. Hence, based on a small set of acquired measurements, the entire signal needs to be reconstructed. The missing samples in compressive sensing generally cannot be recovered using standard interpolation methods due to the complexity of nonstationary signals in real applications. Namely, the interpolation methods such as polynomial fit, cubic spline interpolation, or similar usually assume certain model function, which is mostly inappropriate for time domain signal modeling. Therefore, the compressive sensing reconstruction is formulated in the literature as an optimization problem (rather than interpolation) which reconstructs the signal by finding the sparsest transform domain solution corresponding to the available small set of samples.

In the CS context, we are dealing with a small set of randomly chosen samples of $f(t)$. Let us assume that we have only $M_{A}$ out of $M$ available samples ( $M$ is the total number of signal samples and $\left.M \gg M_{A}\right)$. The vector of available measurements is denoted as $\mathbf{y}$. Now we may write

$$
\mathbf{y}=\boldsymbol{\Phi f}
$$

where $\mathbf{f}$ is original full signal (of length $M$ ) written in the vector form, while $\Phi\left(M_{A} \times M\right)$ is the measurement matrix. The original signal $\mathbf{f}$ can be expressed using the Hermite transform as follows:

$$
\mathbf{f}=\Psi \mathrm{C},
$$

where $\mathbf{C}$ is the vector of Hermite transform coefficients, while $\Psi$ is the inverse Hermite transform matrix of size $M \times N(N \leq$ $M)$ :

$$
\begin{aligned}
& \Psi=\left[\begin{array}{cccc}
\psi_{0}(0) & \psi_{1}(0) & \cdots & \psi_{N-1}(0) \\
\psi_{0}(1) & \psi_{1}(1) & \cdots & \psi_{N-1}(1) \\
\cdots & \cdots & \cdots & \cdots \\
\psi_{0}(M-1) & \psi_{1}(M-1) & \cdots & \psi_{N-1}(M-1)
\end{array}\right], \\
& \mathbf{H}=\frac{1}{M} \\
& {\left[\begin{array}{cccc}
\frac{\psi_{0}(0)}{\left(\psi_{N-1}(0)\right)^{2}} & \frac{\psi_{0}(1)}{\left(\psi_{N-1}(1)\right)^{2}} & \cdots & \frac{\psi_{0}(M-1)}{\left(\psi_{N-1}(M-1)\right)^{2}} \\
\frac{\psi_{1}(0)}{\left(\psi_{N-1}(0)\right)^{2}} & \frac{\psi_{1}(1)}{\left(\psi_{N-1}(1)\right)^{2}} & \cdots & \frac{\psi_{1}(M-1)}{\left(\psi_{N-1}(M-1)\right)^{2}} \\
\cdots & \cdots & \cdots & \cdots \\
\frac{\psi_{N-1}(0)}{\left(\psi_{N-1}(0)\right)^{2}} & \frac{\psi_{N-1}(1)}{\left(\psi_{N-1}(1)\right)^{2}} & \cdots & \frac{\psi_{N-1}(M-1)}{\left(\psi_{N-1}(M-1)\right)^{2}}
\end{array}\right] .}
\end{aligned}
$$


The direct Hermite transform matrix is given by $\mathbf{H}$. In the extended form, (9) can be written as follows:

$$
\begin{gathered}
{\left[\begin{array}{c}
f(0) \\
f(1) \\
\cdots \\
f(M-1)
\end{array}\right]} \\
\underbrace{\left[\begin{array}{cccc}
\psi_{0}(0) & \psi_{1}(0) & \cdots & \psi_{N-1}(0) \\
\psi_{0}(1) & \psi_{1}(1) & \cdots & \psi_{N-1}(1) \\
\cdots & \cdots & \cdots & \cdots \\
\psi_{0}(M-1) & \psi_{1}(M-1) & \cdots & \psi_{N-1}(M-1)
\end{array}\right]}_{\mathbf{f}} \underbrace{\left[\begin{array}{c}
C(0) \\
C(1) \\
\cdots \\
C(N-1)
\end{array}\right]}_{\Psi} .
\end{gathered}
$$

The Hermite basis functions are calculated using the fast recursive realization defined as follows:

$$
\begin{aligned}
& \psi_{0}(t)=\frac{1}{\sqrt[4]{\pi}} e^{-t^{2} / 2}, \\
& \psi_{1}(t)=\frac{\sqrt{2} t}{\sqrt[4]{\pi}} e^{-t^{2} / 2}, \\
& \psi_{i}(t)=t \sqrt{\frac{2}{i}} \Psi_{i-1}(t)-\sqrt{\frac{i-1}{i}} \Psi_{i-2}(t), \quad \forall i \geq 2 .
\end{aligned}
$$

According to (8) and (9), we have

$$
\mathrm{y}=\Phi \Psi \mathrm{C}=\Theta \mathrm{C} .
$$

The reconstructed signal $\mathbf{f}$ can be obtained as a solution of $M_{A}$ linear equations with $M$ unknowns. The system is undetermined and can have infinitely many solutions. Now we assume that the signal is sparse in the Hermite transform domain. It means that the observed signal can be efficiently represented by a very small number $K$ of Hermite expansion coefficients, such that $K<M_{A}$. Therefore, the optimization based mathematical algorithms should be employed to search for the sparsest solution. A near optimal solution is achieved by using the $l_{1}$ norm based minimization as follows:

$$
\begin{aligned}
\min & \|\widehat{\mathbf{C}}\|_{l_{1}} \\
\text { subject to } & \mathbf{y}=\mathbf{\Theta C} \text {, }
\end{aligned}
$$

where $\widehat{\mathbf{C}}$ is the Hermite transform vector of signal $\mathbf{f}$.

In order to solve the previous minimization problem, first we need to calculate the initial Hermite transform using the available set of $M_{A}$ samples with the time support $\Omega: \mathbf{y}=$ $\boldsymbol{\Phi f}=\mathbf{f}(\Omega)$. Therefore, we observe the signal in the following form:

$$
f_{0}(n)= \begin{cases}y(n), & \text { for } n \in \Omega \\ 0, & \text { elsewhere. }\end{cases}
$$

The initial vector of Hermite transform coefficients can be then calculated using $N$ Hermite functions $(N \leq M)$ as follows:

$$
\mathbf{C}_{0}=\mathbf{H}_{\Omega} \mathbf{y}
$$

where $\mathbf{H}_{\Omega}$ contains only the columns of $\mathbf{H}$ that correspond to instants $n \in \Omega$. Alternatively, we can write

$$
C_{0}(i)=\frac{1}{M} \sum_{n=0}^{M-1} \frac{\psi_{i}(n)}{\left(\psi_{M-1}(n)\right)^{2}} f_{0}(n), \quad i=1, \ldots, N
$$

In order to determine the signal support in the Hermite transform domain, the initial vector of the Hermite transform coefficients $\mathbf{C}_{0}$ is compared by the threshold $T$ :

$$
\mathbf{k}=\arg \left\{\mathbf{C}_{0}>T\right\} .
$$

The exact values of Hermite coefficients at positions selected in vector $\mathbf{k}$ are obtained as a solution of the CS problem:

$$
\Theta_{\mathrm{CS}} \mathrm{C}_{0}=\mathbf{y}
$$

The CS matrix $\boldsymbol{\Theta}_{\mathrm{CS}}$ is obtained from the inverse Hermite transform matrix $\Theta_{\mathrm{CS}}=\Psi(\Omega, \mathbf{k})$, using columns that correspond to frequencies $\mathbf{k}$ and rows corresponding to measurements with support $\Omega$. The system is solved in the least square sense as follows:

$$
\mathbf{C}=\left(\boldsymbol{\Theta}_{\mathrm{CS}}^{*} \boldsymbol{\Theta}_{\mathrm{CS}}\right)^{-1} \boldsymbol{\Theta}_{\mathrm{CS}}^{*} \mathbf{y},
$$

where $(*)$ denotes the conjugate transpose operation.

Analysis of Components Reconstruction. Let us consider the isometry property of Hermite transform matrix $\Psi$ :

$$
\begin{aligned}
\| \Psi \mathbf{C} & \left\|_{2}^{2}=\right\| f \|_{2}^{2}=|f(0)|^{2}+\cdots+|f(N-1)|^{2} \\
= & \left|\sum_{k=0}^{N-1} C(k) \psi_{k}(0)\right|^{2}+\cdots+\left|\sum_{k=0}^{N-1} C(k) \psi_{k}(N-1)\right|^{2} \\
= & \sum_{k=0}^{N-1} C(k)^{2} \sum_{n=0}^{N-1} \psi_{k}(n)^{2} \\
& +\sum_{k_{1}=0}^{N-2} \sum_{k_{2}=k_{1}+1}^{N-1} 2 C\left(k_{1}\right) C\left(k_{2}\right) \sum_{n=0}^{N-1} \psi_{k_{1}}(n) \psi_{k_{2}}(n) .
\end{aligned}
$$

The previous equation can be written as follows:

$$
\begin{aligned}
& \|\boldsymbol{\Psi} \mathbf{C}\|_{2}^{2}=\left((C(0))^{2} \sum_{n=0}^{N-1}\left(\psi_{0}(n)\right)^{2}+\cdots\right. \\
& \left.+(C(N-1))^{2} \sum_{n=0}^{N-1}\left(\psi_{N-1}(n)\right)^{2}\right) \\
& +\sum_{k_{1}=0}^{N-2} \sum_{k_{2}=k_{1}+1}^{N-1} 2 C\left(k_{1}\right) C\left(k_{2}\right) \sum_{n=0}^{N-1} \psi_{k_{1}}(n) \psi_{k_{2}}(n) .
\end{aligned}
$$

Now, observe the first term on the right side given by $P=C(0)^{2} \sum_{n=0}^{N-1}\left(\psi_{0}(n)\right)^{2}+\cdots+C(N-1)^{2} \sum_{n=0}^{N-1}\left(\psi_{N-1}(n)\right)^{2}$, particularly the sum of squared values of different Hermite functions. Figure 1 illustrates the sum of squared values of different Hermite functions calculated in the zeros of Hermite 


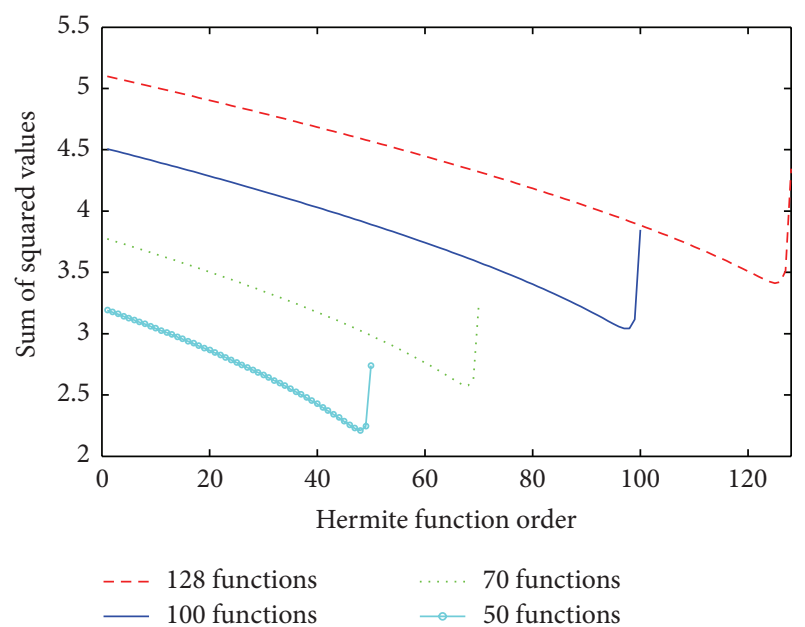

FIGURE 1: Sum of squared values of Hermite functions for different $N$.

polynomials for $N=(128,100,70$ and 50). Unlike in the case of the Fourier transform basis, where the sum of absolute values of complex exponential basis would be constant for any frequency $k$, from Figure 1 we can note an approximate low-pass characteristic of the curves, meaning that the lowerorder coefficients are favored compared to the higher order coefficients. Consequently, when applying the threshold for components detection, it would be easier to detect a set of low-order coefficients than the high-order ones.

\section{Introducing the Short-Time Hermite Transform and Short-Time Combined Transform}

4.1. Short-Time Hermite Transform. Let us assume that $N=$ $M$ in (10) and define an $M \times M$ Hermite matrix:

$$
\begin{aligned}
\mathbf{H}_{M} & =\frac{1}{M} \\
& {\left[\begin{array}{cccc}
\frac{\psi_{0}(0)}{\left(\psi_{M-1}(0)\right)^{2}} & \frac{\psi_{0}(1)}{\left(\psi_{M-1}(1)\right)^{2}} & \cdots & \frac{\psi_{0}(M-1)}{\left(\psi_{M-1}(M-1)\right)^{2}} \\
\frac{\psi_{1}(0)}{\left(\psi_{M-1}(0)\right)^{2}} & \frac{\psi_{1}(1)}{\left(\psi_{M-1}(1)\right)^{2}} & \cdots & \frac{\psi_{1}(M-1)}{\left(\psi_{M-1}(M-1)\right)^{2}} \\
\ldots & \ldots & \cdots & \cdots \\
\frac{\psi_{M-1}(0)}{\left(\psi_{M-1}(0)\right)^{2}} & \frac{\psi_{M-1}(1)}{\left(\psi_{M-1}(1)\right)^{2}} & \cdots & \frac{\psi_{M-1}(M-1)}{\left(\psi_{M-1}(M-1)\right)^{2}}
\end{array}\right] . }
\end{aligned}
$$

The short-time Hermite transform (STHT) can be defined as a composition of Hermite transform matrices whose size is defined by the window width. Without loss of generality, we may assume the nonoverlapping unit rectangular windows (with the width of $M$ samples). The total transform matrix for the STHT can be created as follows:

$$
\mathbf{W}=\mathbf{I}_{L / M} \otimes \mathbf{H}_{M}=\left[\begin{array}{cccc}
\mathbf{H}_{M} & \mathbf{0} & \cdots & \mathbf{0} \\
\mathbf{0} & \mathbf{H}_{M} & \cdots & \mathbf{0} \\
\cdots & \cdots & \cdots & \mathbf{0} \\
\mathbf{0} & \mathbf{0} & \cdots & \mathbf{H}_{M}
\end{array}\right],
$$

where I is identity matrix of size $(L / M \times L / M), \mathbf{0}$ is $M \times M$ zero matrix, and $L$ is the total length of the signal, while $\otimes$ denotes the Kronecker product. The STHT can be defined using transform matrix $\mathbf{W}$ as follows:

$$
\mathrm{STHT}=\mathrm{Wf}=\mathrm{W} \Psi_{L} \mathrm{C},
$$

where $\mathbf{f}$ is a time domain signal vector of size $L \times 1$ and $\mathbf{C}$ is a Hermite transform vector of size $L \times 1$, while STHT is a column vector containing all STHT vectors $\mathbf{S T H T}_{M_{i}}\left(m_{i}\right)$, $i=0,1, \ldots, P$ ( $P$ is the number of windows). Furthermore we may write

$$
\text { STHT }=\text { AC, }
$$

where $\mathbf{A}=\mathbf{W} \Psi_{L}$ is matrix of size $L \times L$ that maps the global transform domain information in $\mathbf{C}$ into local information in STHT. In the case of windows with variable length over time (time-varying windows), the smaller matrices within $\mathbf{W}$ will be of different sizes. Particularly, for a set of $P$ rectangular windows of sizes $M_{1}, M_{2}, \ldots, M_{P}$ instead of $\mathbf{H}_{M}$ we will have $\mathbf{H}_{M_{1}}, \mathbf{H}_{M_{2}}, \ldots, \mathbf{H}_{M_{P}}$.

In the context of compressive sensing, the STHT allows us to define two types of CS problem. A common CS problem is defined by assuming that the missing samples appear in the time domain, while the sparsity is exploited in the transform domain. However, the missing samples may also appear in the STHT domain, after applying certain filter forms such as the $L$-estimate filtering in the presence of strong noisy pulses. The two CS optimization problems are discussed in the sequel.

(1) Assume that CS procedure is done in the time domain, such that the measurements are taken from each windowed signal part: $\mathbf{y}_{i}=\mathbf{f}_{M_{i}}(\Omega)$. Based on the definitions given in Section 3 , the minimization problem is given by

$$
\begin{aligned}
\min & \left\|\mathbf{C}_{i}\right\|_{l 1} \\
\text { subject to } & \mathbf{y}_{i}=\Psi_{M}^{\Omega} \mathbf{C}_{i},
\end{aligned}
$$

for $i=1,2, \ldots, P$, where $P$ is the total number of windows, while $\Psi_{M}$ is the inverse Hermite transform of size $M \times M$, where only the rows corresponding to the set of positions $\Omega$ are kept.

(2) Let us observe the missing values in the STHT domain, where the measurements vector is denoted by STHT $_{\text {CS }}$. The CS problem formulation is defined using the linear relationship (26). The CS matrix, denoted by $\mathbf{A}_{\mathrm{CS}}$, is formed by omitting the rows from $\mathbf{A}$ corresponding to the positions of missing values in $\mathbf{S T H T}_{\mathrm{CS}}$. Then, a simple linear relationship can be established between the reduced 


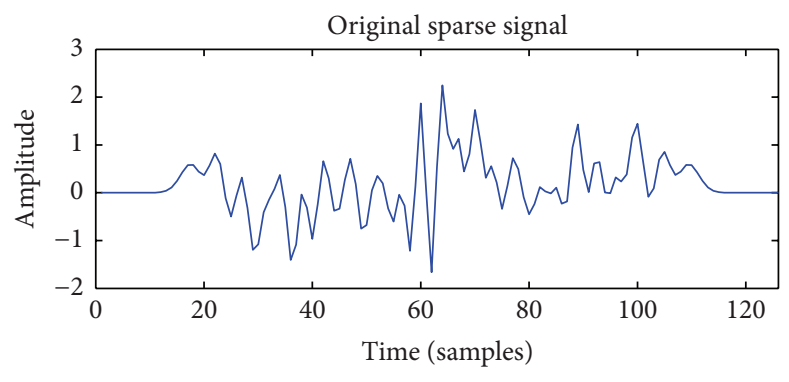

(a)

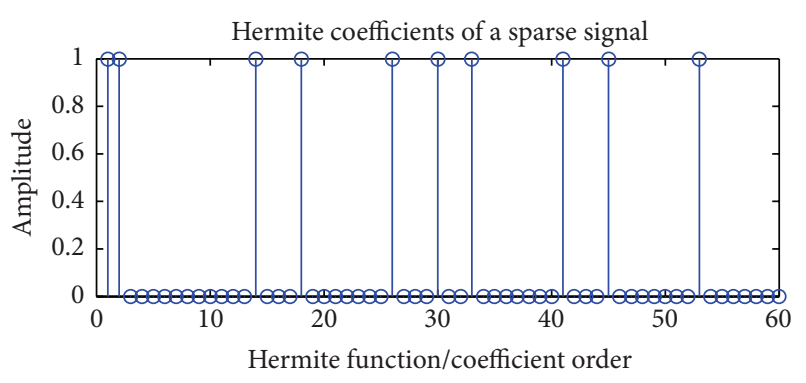

(b)

FIgURE 2: The original signal: (a) time domain representation, (b) Hermite transform of signal calculated using $N=60 \mathrm{Hermite}$ functions.

observations in STHT $_{\mathrm{CS}}$ and the sparse Hermite transform vector $\mathbf{C}$ (corresponding to the entire signal). Hence, the CS problem is given in the following form:

$$
\begin{aligned}
\min & \|\mathbf{C}\|_{1} \\
\text { subject to } & \text { STHT }_{\mathrm{CS}}=\mathbf{A}_{\mathrm{CS}} \mathbf{C} .
\end{aligned}
$$

It means that the missing samples in the STHT can be reconstructed such as to provide the best concentrated $\mathbf{C}$. The above CS optimization problem can be solved using various existing CS reconstruction algorithms.

4.2. Short-Time Combined Transform. On the basis of the STHT, we can also define the short-time combined transform as follows:

$$
\mathbf{X}=\mathbf{Z f}
$$

where the transform matrix $\mathbf{Z}$ is made as a combination of Hermite transform matrices and other transforms:

$$
\mathbf{Z}=\left[\begin{array}{cccc}
\mathbf{Z}_{M_{1}} & \mathbf{0} & \cdots & \mathbf{0} \\
\mathbf{0} & \mathbf{Z}_{M_{2}} & \cdots & \mathbf{0} \\
\cdots & \cdots & \cdots & \mathbf{0} \\
\mathbf{0} & \mathbf{0} & \cdots & \mathbf{Z}_{M_{P}}
\end{array}\right]
$$

where $\mathbf{Z}_{M_{i}}$ can be either the $M_{i} \times M_{i}$ Hermite transform or some other suitable transform matrix of the same size. For instance, we may observe one example of the combination of Hermite transform and Fourier transform as a special case:

$$
\mathbf{Z}=\left[\begin{array}{cccc}
\mathbf{H}_{M} & \mathbf{0} & \cdots & \mathbf{0} \\
\mathbf{0} & \mathbf{F}_{M} & \cdots & \mathbf{0} \\
\cdots & \cdots & \mathbf{F}_{M} & \mathbf{0} \\
\mathbf{0} & \mathbf{0} & \cdots & \mathbf{H}_{M}
\end{array}\right]
$$

The combined matrix $\mathbf{Z}$ is thus composed of Hermite transform matrix $\mathbf{H}_{M}$ of size $M \times M$, two sequential Fourier transform matrices $\mathbf{F}_{M}$ of size $M \times M$, and again one Hermite transform matrix of the same size. In the case of windows with variable length over time, the short-time combined transform can be written as:

$$
\left[\begin{array}{c}
\mathbf{X}_{M_{1}} \\
\mathbf{X}_{M_{2}} \\
\cdots \\
\mathbf{X}_{M_{P}}
\end{array}\right]=\left[\begin{array}{cccc}
\mathbf{Z}_{M_{1}} & \mathbf{0} & \cdots & \mathbf{0} \\
\mathbf{0} & \mathbf{Z}_{M_{2}} & \cdots & \mathbf{0} \\
\cdots & \cdots & \cdots & \mathbf{0} \\
\mathbf{0} & \mathbf{0} & \cdots & \mathbf{Z}_{M_{P}}
\end{array}\right]\left[\begin{array}{c}
\mathbf{f}_{M_{1}} \\
\mathbf{f}_{M_{2}} \\
\cdots \\
\mathbf{f}_{M_{P}}
\end{array}\right],
$$

where $\mathbf{f}_{M_{1}}$ represents the vector containing first $M_{1}$ samples in $\mathbf{f}$ and vector $\mathbf{f}_{M_{2}}$ contains the next $M_{2}$ samples from $\mathbf{f}$, while $\mathbf{f}_{M_{P}}$ contains the last $M_{P}$ samples from $\mathbf{f}$ such that $M_{1}+M_{2}+$ $\cdots+M_{P}=L$.

We can again observe the set of measurements corresponding to different signal parts: $\mathbf{y}_{i}=\mathbf{f}_{M_{i}}(\Omega)$, where $\boldsymbol{\Theta}_{M_{i}}=$ $\operatorname{inv}\left(\mathbf{Z}_{M_{i}}\right)$. Therefore, the CS minimization problem is given by

$$
\begin{aligned}
\min & \left\|\mathbf{X}_{M_{i}}\right\|_{1} \\
\text { subject to } & \mathbf{y}_{i}=\Theta_{M_{i}}^{\Omega} \mathbf{X}_{M_{i}},
\end{aligned}
$$

for $i=1,2, \ldots, P$, where $\Omega$ in superscript denotes that we use only the rows corresponding to available samples defined by the set $\Omega$. The inverse transform denoted by $\Theta_{M_{\text {i }}}^{\Omega}$ can be changed in each window depending on the signal characteristics.

\section{Experimental Evaluation}

Example 1. In order to illustrate efficiency of the proposed method, let us observe a signal that is sparse in the Hermite transform domain. The time domain signal is shown in Figure 2(a). The Hermite transform of the observed signal consists of ten components with unit amplitudes, as shown in Figure 2(b). The available number of samples is $60 \%$ of the total signal length, while the maximal order of Hermite functions used in the expansion is limited to $N=60$.

After calculating the initial Hermite transform using available signal samples, the threshold is applied to select signal support in the Hermite domain. The threshold is set empirically to $T=\alpha \max \{\mathbf{C}\}, \alpha=0.4$, after performing a large number of experiments. Moreover, the results are not very sensitive on threshold settings, and even lower thresholds (e.g., $\alpha=0.3, \alpha=0.2$ ) can be used, because all additional 


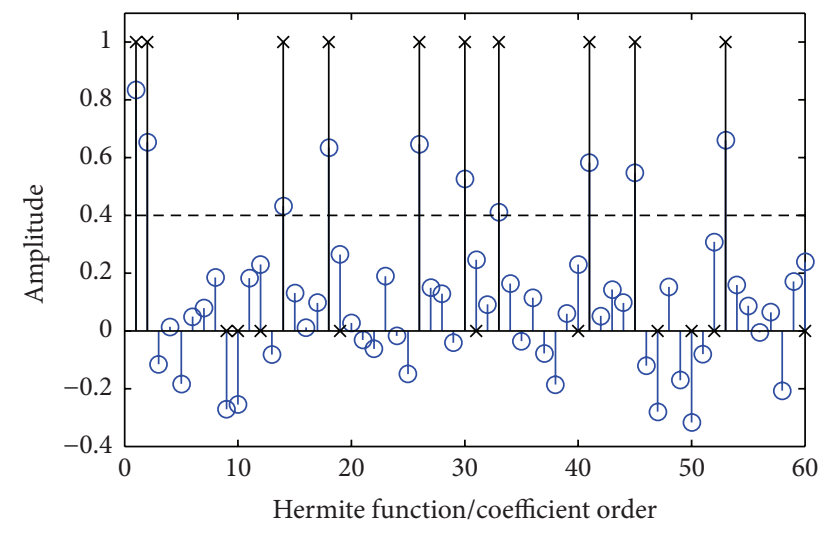

FIGURE 3: Hermite coefficients of signal with missing samples -O, and the reconstructed Hermite coefficients $-x$.

(false) components selected by such a threshold would obtain their true zero values afterwards. The suitable threshold can be even theoretically derived (under certain assumptions). Namely, the effects caused by missing samples in time domain can be modeled by the noise in the Hermite transform domain. The noise can be described using two random variables: the first one corresponds to the noise appearing at nonsignal components (noise alone), while the second one corresponds to the noise appearing at the signal components positions. The two random variables can be described by the corresponding mean values and variances that could be further employed to define the threshold level. Namely, with certain probability, we may define the threshold value that is just above the level of noise components. Consequently, such a threshold would select only the signal components determining the signal support used in the reconstruction procedure. Since the threshold derivation would require significant space and analyses, it could be a topic of some further work.

The selected components in the Hermite transform domain are shown in Figure 3. Using the detected positions of components, the exact values of Hermite coefficients are calculated according to (20) (Figure 3 ). The reconstructed signal and the absolute error between original and reconstructed signal are shown in Figures 4(a) and 4(b), respectively. For the comparison, the reconstruction based on the Fourier transform is considered. However, it is shown that the Fourier transform based reconstruction cannot be used in this case, because it produces a significant error (Figure 4(c), 10 largest Fourier transform components are used for reconstruction). The reason is in the fact that the signals that are sparse in Hermite transform domain do not exhibit sparsity in the Fourier transform domain (or some other transform domains). This means that the exact results, in this case, can be obtained only using the Hermite transform providing the sparse signal representation. Similar conclusion holds for other transform domains where the signal is not sparse (Fractional Fourier, Wavelet, etc.).

Example 2. In this example we have observed different sparse signals (having 10 components) in the Hermite domain through the 1000 repetitions of the previously described procedure. The aim is to test how the accuracy of the proposed method changes for different number of missing samples. Namely, the number of missing samples was increased in steps of $10 \%$ starting from zero up to $80 \%$ of missing samples. For each instance on the $y$-axes, we performed 1000 iterations with different 10-component signals, and the mean square errors between original and reconstructed signals are calculated and shown in Figure 5(a). We can observe that, up to $70 \%$ of missing samples, the MSE is still low, but it starts to increase significantly afterwards (for values higher than $70 \%$ ). This means that the reliable reconstruction cannot be guaranteed for such a large number of missing samples (such as $80 \%$ of missing samples).

Additionally, the proposed approach is tested in the presence of external additive Gaussian noise. Namely, the MSE is calculated for different values of SNR (for each SNR, we assume 1000 realizations of random noise). The results are presented in Figure 5(b), showing that the proposed approach can be efficiently used for SNR $>8 \mathrm{~dB}$. For SNR $<8 \mathrm{~dB}$ the MSE starts to increase rapidly.

Example 3. This example illustrates the concept of combined Hermite-Fourier transform and CS reconstruction. Observe the signal with the total length of $L=32$ samples composed of two parts: first part exhibits sparsity in the Hermite transform domain with $K=2$ components (HT components) at positions 7 and 12 with amplitudes 0.8 and 0.65 ; second part exhibits sparsity in the Fourier transform domain with $K=2$ components (FT components) at positions -3 and 4 with amplitudes 1 and 0.8 , respectively. Now consider the compressive sensing scenario with only $50 \%$ of samples available (original full length signal and available measurements are shown in Figure 6). The original short-time combined transform obtained using windows width equal to 16 samples is shown in Figure 7(a), where the white fields represent 0 value. Thus, the signal represented by a full set of samples is sparse meaning that it is fully concentrated on a few nonzero components. In the case when we deal with $50 \%$ of missing samples, the corresponding short-time combined transform is shown in Figure 7(b). Note that in this case the sparsity is disturbed due to the missing samples. Instead of zero values at nonsignal position, the noise appears as a consequence of missing samples.

The threshold based components selection and signal reconstruction (in analogy to (18) and (20)) is applied to both parts of the signal, FT components, and HT components (Figures $8(\mathrm{a})$ and $8(\mathrm{~b})$, resp.). Based on the identified positions of components, the exact signal reconstruction is done using the procedure presented in Section 3. The reconstructed signal components are shown in Figure 9.

\section{Conclusion}

The possibility of using the Hermite transform in compressive sensing applications was explored in this work. The compressive sensing setup and signal reconstruction approach in the Hermite transform domain were defined. A simple and 


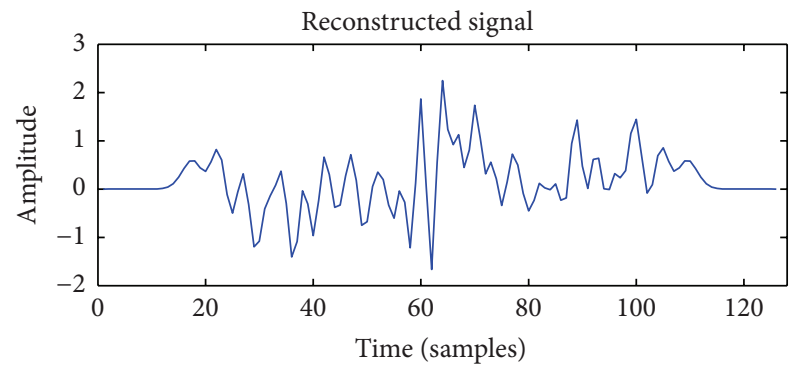

(a)

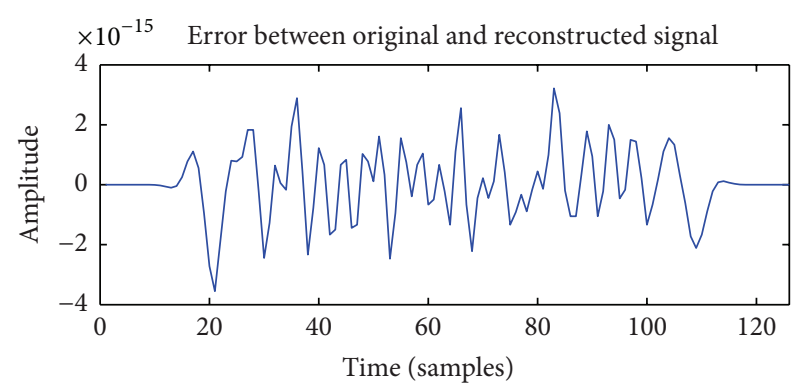

(b)

Reconstructed signal and error between original and reconstructed signal

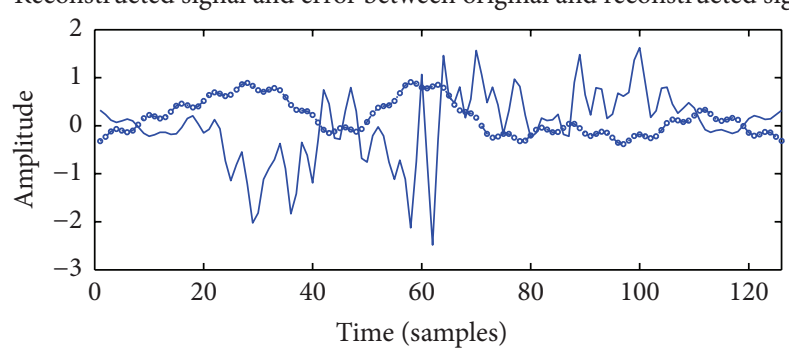

(c)

FIGURE 4: The reconstructed signal: (a) time domain representation, (b) the absolute error between original and reconstructed signal, (c) for comparison: reconstructed signal based on the Fourier transform reconstruction approach (- $\circ$ ) and the corresponding error (solid line).

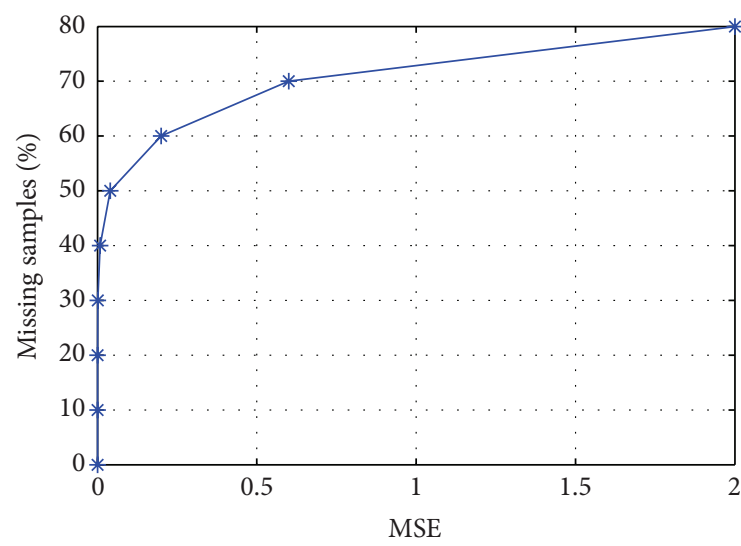

(a)

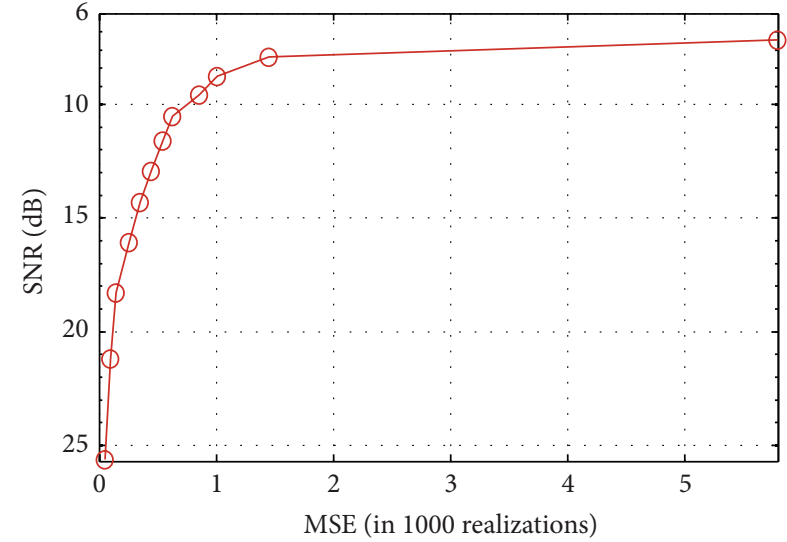

(b)

FIGURE 5: (a) MSE calculated for 1000 realizations and for different number of missing samples, (b) MSE calculated for different values of SNR in 1000 realizations of external noise.

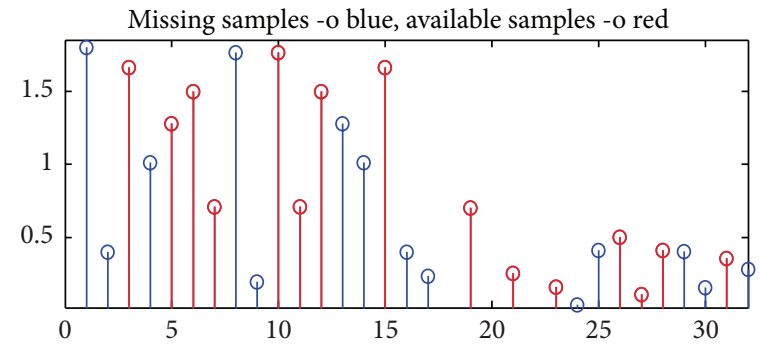

FIGURE 6: Missing samples and available measurements in time domain. 


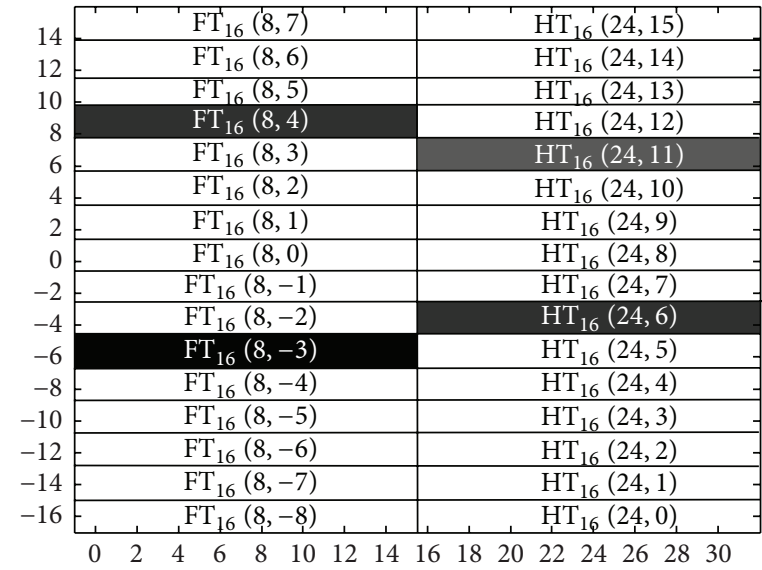

(a)

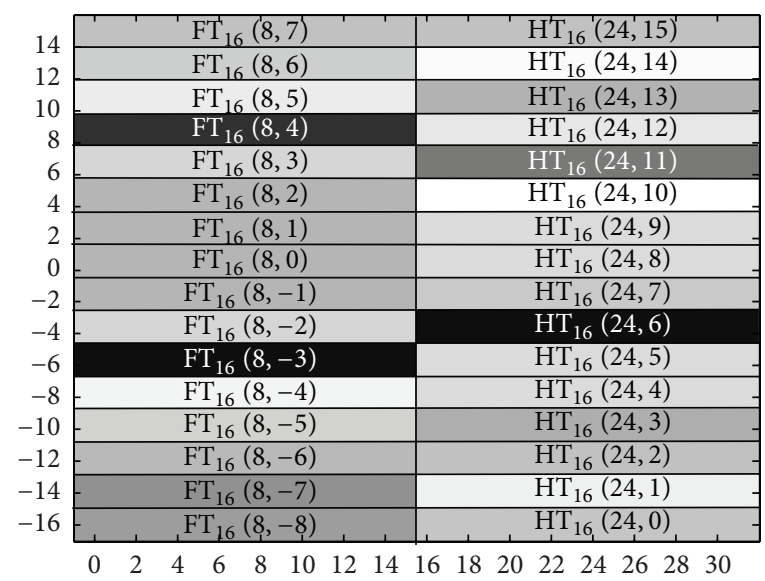

(b)

FIGURE 7: (a) Original short-time combined transform of full length signal (FT: Fourier transform, HT: Hermite transform), (b) short-time combined transform of available measurements.

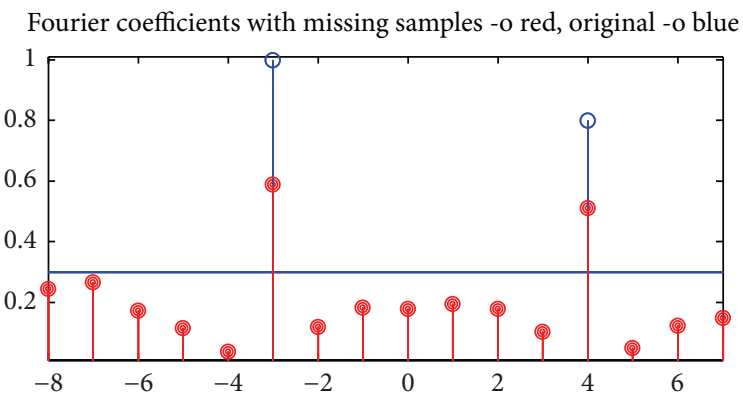

(a)
Hermitian coefficients with missing samples -o red, original -o blue

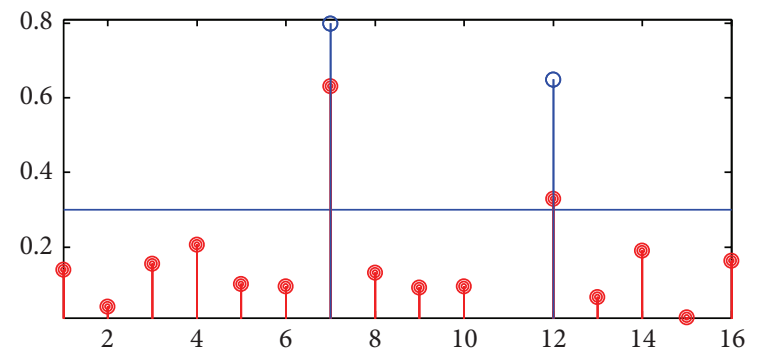

(b)

FIGURE 8: Selecting the components of interest by applying threshold to the following: (a) FT components and (b) HT components.
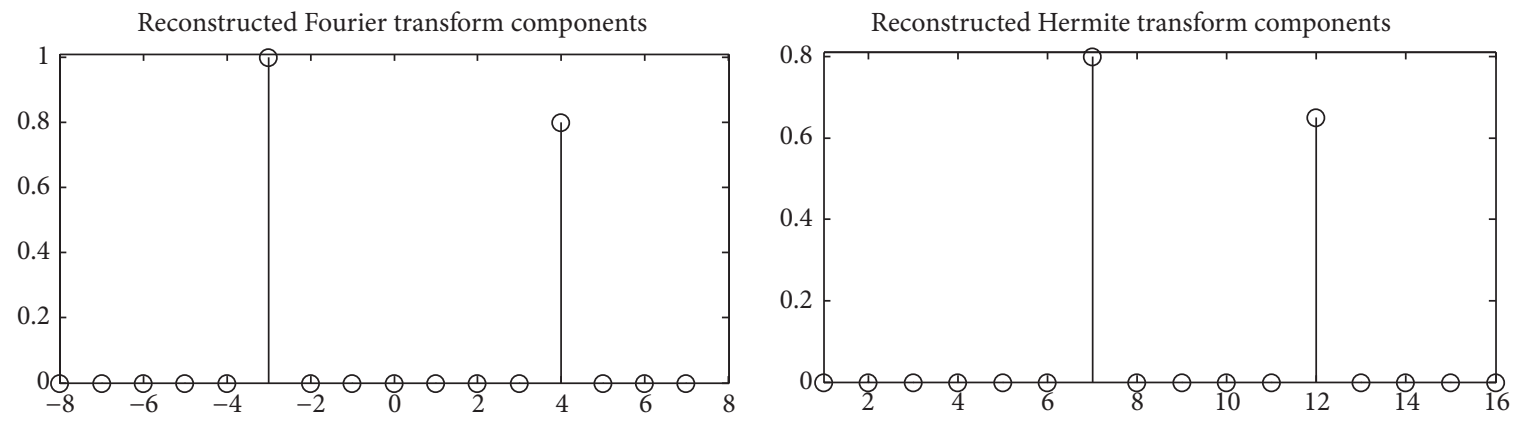

FIGURE 9: Reconstructed signal components.

fast procedure for the total reconstruction of signals using selected Hermite transform coefficients provides the results very close to the original signal even when we deal with significant missing information. It is important to emphasize that the proposed concept in the Hermite transform domain can be also combined with other known compressive sensing solvers. The entire concept is generalized and extended by defining the short-time Hermite transform as well as the short-time combined transform. These two transforms open more possibilities to apply the compressive sensing approach in different scenarios.

\section{Conflict of Interests}

The authors declare that there is no conflict of interests regarding the publication of this paper.

\section{Acknowledgment}

This work is supported by the Montenegrin Ministry of Science, Project Grant "New ICT Compressive Sensing Based Trends Applied to: Multimedia, Biomedicine and Communications" (ACRONYM: CS-ICT). 


\section{References}

[1] A. Sandryhaila, S. Saba, M. Püschel, and J. Kovacevic, "Efficient compression of QRS complexes using hermite expansion," IEEE Transactions on Signal Processing, vol. 60, no. 2, pp. 947-955, 2012.

[2] M. Lagerholm, G. Peterson, G. Braccini, L. Edenbrandt, and L. L. Sörnmo, "Clustering ECG complexes using hermite functions and self-organizing maps," IEEE Transactions on Biomedical Engineering, vol. 47, no. 7, pp. 838-848, 2000.

[3] B. Beliczynski, "Approximation of functions by multivariable hermite basis: a hybrid method," in Adaptive and Natural Computing Algorithms, vol. 6593 of Lecture Notes in Computer Science, pp. 130-139, Springer, 2011.

[4] Y. K. Alp and O. Arıkan, "Time-frequency analysis of signals using support adaptive Hermite-Gaussian expansions," Digital Signal Processing, vol. 22, no. 6, pp. 1010-1023, 2012.

[5] A. Krylov and D. Kortchagine, "Fast Hermite projection method," in Proceedings of the International Conference on Image Analysis and Recognition (ICIAR '06), pp. 329-338, Póvoa de Varzim, Portugal, September 2006.

[6] D. Kortchagine and A. Krylov, "Projection filtering in image processing," in Proceedings of the International Conference on Computer Graphics and Applications, pp. 42-45, Moscow, Russia, August-September 2000.

[7] I. Orovi, S. Stankovi, T. Chau, C. M. Steele, and E. Sejdić, “Timefrequency analysis and hermite projection method applied to swallowing accelerometry signals," EURASIP Journal on Advances in Signal Processing, vol. 2010, Article ID 323125, 2010.

[8] R. G. Baraniuk, "Compressive sensing," IEEE Signal Processing Magazine, vol. 24, no. 4, pp. 118-124, 2007.

[9] D. L. Donoho, "Compressed sensing," IEEE Transactions on Information Theory, vol. 52, no. 4, pp. 1289-1306, 2006.

[10] E. J. Candes, J. Romberg, and T. Tao, "Robust uncertainty principles: exact signal reconstruction from highly incomplete frequency information," IEEE Transactions on Information Theory, vol. 52, no. 2, pp. 489-509, 2006.

[11] D. Vukobratovic and A. Pizurica, "Compressed sensing using sparse adaptive measurements," in Proceedings of the Symposium on Information Theory in the Benelux (SITB '14), Eindhoven, The Netherlands, May 2014.

[12] D. Angelosante, G. B. Giannakis, and E. Grossi, "Compressed sensing of time-varying signals," in Proceedings of the 16th International Conference on Digital Signal Processing (DSP '09), pp. 1-8, Santorini-Hellas, Greece, July 2009.

[13] L. Stankovic, S. Stankovic, and M. Amin, "Missing samples analysis in signals for applications to L-estimation and compressive sensing," Signal Processing, vol. 94, no. 1, pp. 401-408, 2014.

[14] S. Stanković, I. Orović, and L. Stanković, "An automated signal reconstruction method based on analysis of compressive sensed signals in noisy environment," Signal Processing, vol. 104, pp. 4350, 2014.

[15] S. Stanković, L. Stanković, and I. Orović, "Relationship between the robust statistics theory and sparse compressive sensed signals reconstruction," IET Signal Processing, vol. 8, no. 3, pp. 223-229, 2014. 


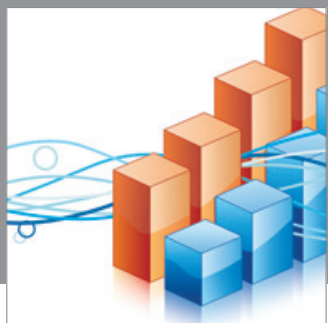

Advances in

Operations Research

mansans

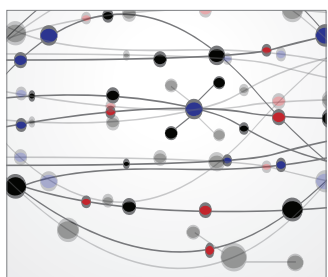

The Scientific World Journal
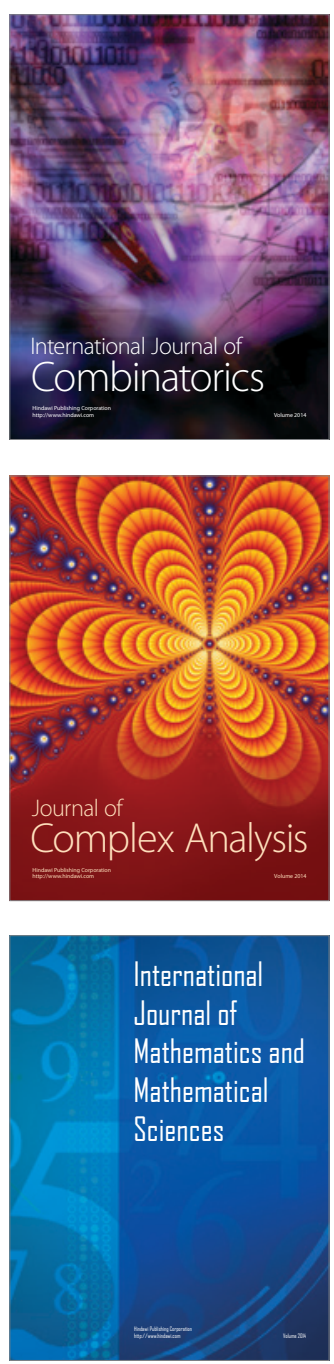
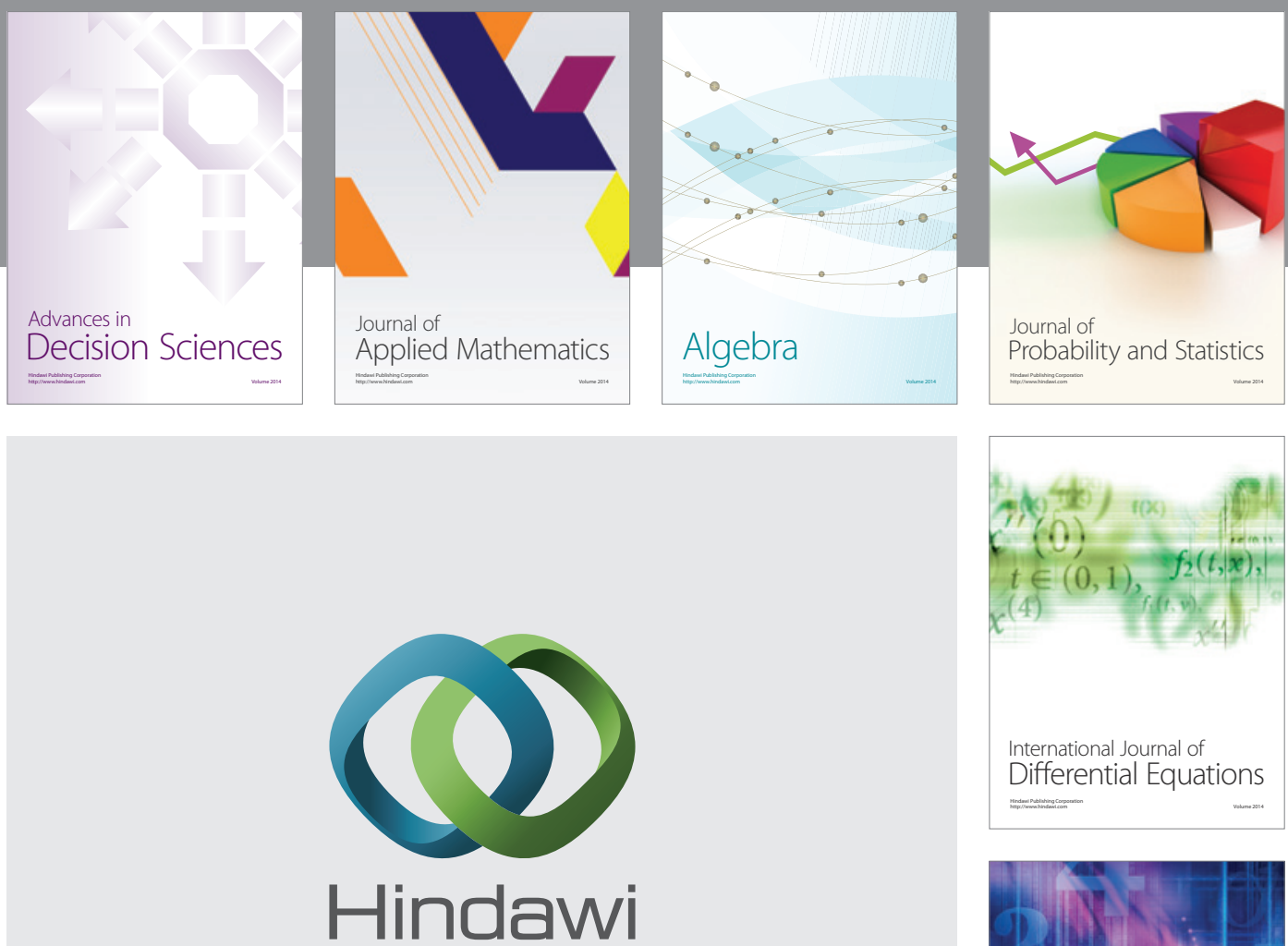

Submit your manuscripts at http://www.hindawi.com
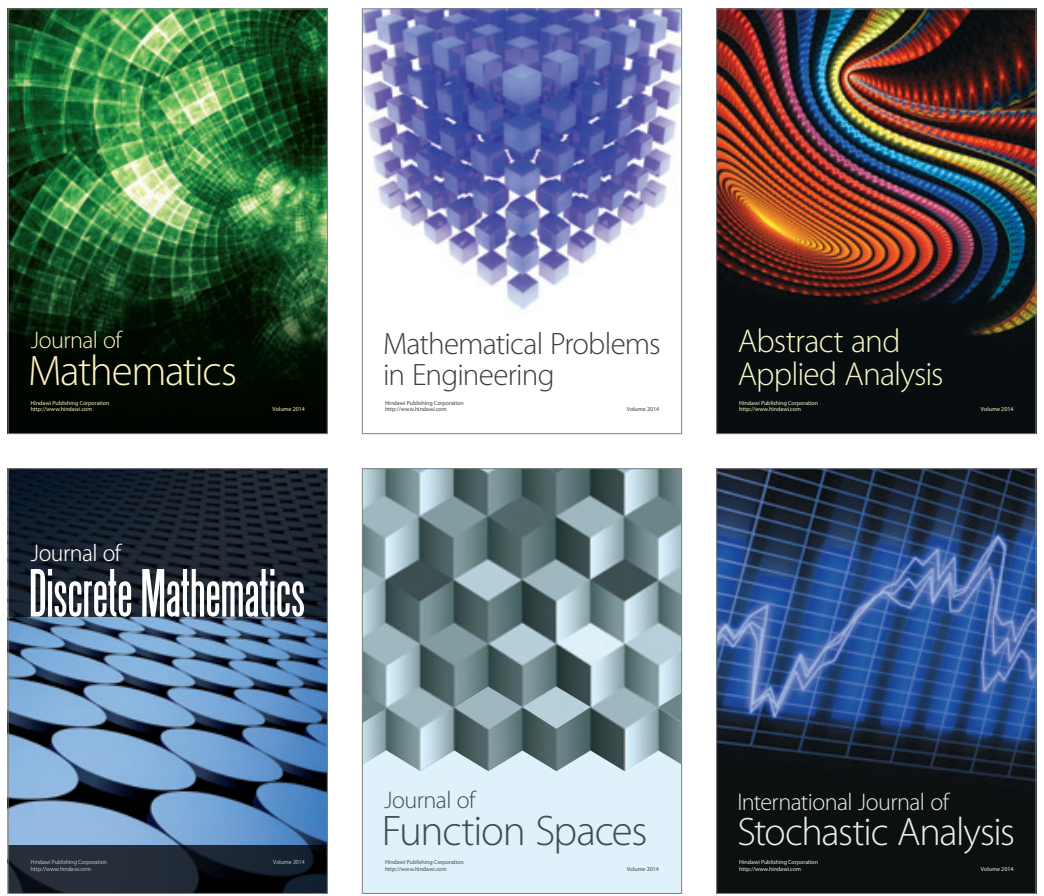

Journal of

Function Spaces

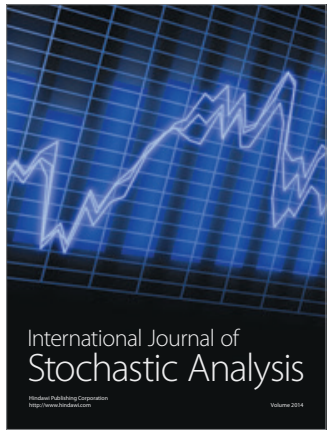

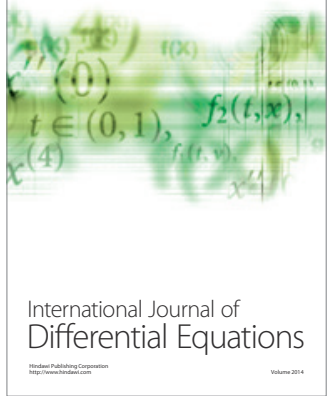
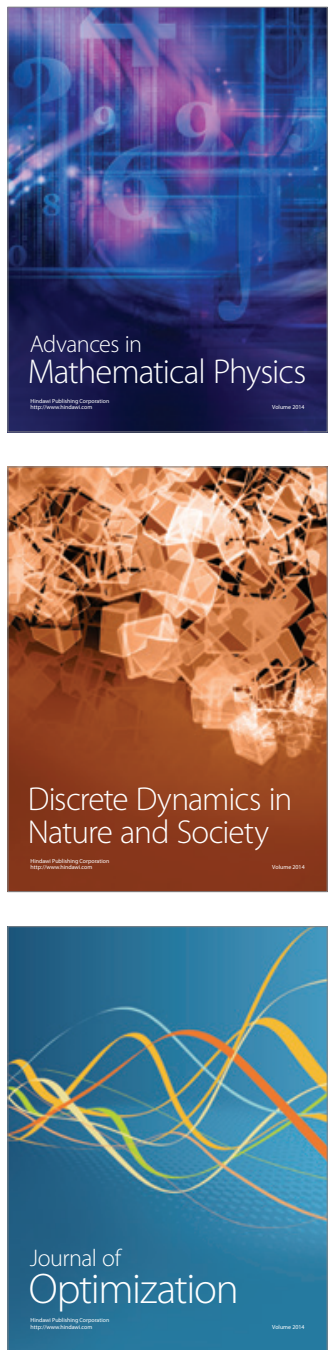